\title{
mHealth: Using Mobile Technology to Support Healthcare
}

\author{
Senanu Okuboyejo1, Omatseyin Eyesan¹
}

1. Covenant University, Department of Computer and Information Sciences, Nigeria

\begin{abstract}
Adherence to long-term therapy in outpatient setting is required to reduce the prevalence of chronic diseases such as HIV/AIDS, Diabetes, Tuberculosis and Malaria. This paper presents a mobile technology-based medical alert system for outpatient adherence in Nigeria. The system makes use of the SMS and voice features of mobile phones. The system has the potential of improving adherence to medication in outpatient setting by reminding patients of dosing schedules and attendance to scheduled appointments through SMS and voice calls. It will also inform patients of benefits and risks associated with adherence. Interventions aimed at improving adherence would provide significant positive return on investment through primary prevention (of risk factors) and secondary prevention of adverse health outcomes.

Keywords: medication adherence; chronic diseases; mobile technology; SMS; Voice; Nigeria; health care.

Correspondence: sena.okuboyejo@covenantuniversity.edu.ng

DOI: 10.5210/ojphi.v5i3.4847

Copyright @2014 the author(s)

This is an Open Access article. Authors own copyright of their articles appearing in the Online Journal of Public Health Informatics. Readers may copy articles without permission of the copyright owner(s), as long as the author and OJPHI are acknowledged in the copy and the copy is used for educational, not-for-profit purposes.
\end{abstract}

\section{Introduction}

The most common contributors to the disease burden in Nigeria are Malaria, Tuberculosis (TB) and HIV/AIDS [1]. These diseases are chronic, infectious/non-infectious, and highly prevalent. The current goals and objectives of the health sector include: reduction of the disease burden from HIV/AIDS, control and eradication of malaria and tuberculosis (which are prevalent and chronic). Chronic diseases are diseases which have one or more of the following characteristics: they are permanent, leave residual disability, are caused by non-reversible pathological alteration, require special training of the patient for rehabilitation, or may be expected to require a long period of supervision, observation or care [2]. Most of the cares needed for chronic conditions are based on patient self-management (usually requiring complex multi-therapies) coupled with strict adherence to medication and will require health system support.

Adherence to long-term therapy in outpatient setting is required to reduce prevalence of these diseases. Adherence is generally described as the extent to which patients take medications as prescribed by their health care providers [3]. Rates of adherence for individual patients are usually reported as the percentage of the prescribed doses of the medication actually taken by the patient over a specified period. Some investigators have further refined the definition of 
adherence to include data on dose taking (taking the prescribed number of pills each day) and the timing of doses (taking pills within a prescribed period). Interventions aimed at improving adherence would provide significant positive return on investment through primary prevention (of risk factors) and secondary prevention of adverse health outcomes [4]. Health outcomes cannot be accurately assessed if they are measured predominantly by resource utilization and efficacy of interventions. Poor adherence to long-term therapies severely compromises the effectiveness of treatment making this a critical issue in population health both from the perspective of quality of life and of health economics.

\subsection{Mobile Technology for Medication Adherence}

Health challenges have been found to be a significant barrier to development in Nigeria. The vulnerability of Nigeria's rural population is summarized with the following reasons: epidemics, late diagnosis of ailment, lack of good health care infrastructure and delay in transport time to urban health care facilities, and inexperienced primary health-care providers in rural areas. ICT diffusion in health care offers the potential to address these concerns and to save the patient extra costs associated with treatment, such as travel and other living expenses. The latest subscriber data released by the Nigerian Communications Commission (NCC) has shown that Nigeria's telecommunications industry's teledensity has hit 73.12 per cent as at the end of June 2012, while the total number of mobile connected lines in the country has climbed to 136.04 million. This rise validates the report by the Association of Worldwide Mobile Phone Operators that Africa (of which Nigeria is a major player) tops the continents using mobile phones in the world. Teledensity is the number of landline telephones in use for every 100 individuals living within an area [5].

The explosive growth and deep penetration of mobile communications in these areas, provides millions of rural dwellers access to reliable technology for communication and data transfer. This growing ubiquity of mobile phones is a promise of the use of mobile technologies for providing mobile health interventions. One advantage, however, of telephones with respect to medication adherence in chronic care models is its ability to create a multi-way interaction between patient and health care provider(s) and thus facilitates the dynamic nature of this relationship [6].

The need to improve and provide efficient health services has resulted in the increased use of information and communication technology-based solutions in the healthcare sector. The use of text messaging in health has garnered increasing attention as a means to track disease outbreaks, monitor patient treatment, diagnose patients, educate patients, collect and transmit data through basic mobile phones. Various Studies have highlighted the potential of mobile phones to disseminate public health information and mobilize attendance to vaccination programs particularly in developing countries as well as to manage the treatment of diabetes in Scotland [7]. Recently, the Patient Care Messaging Service for Pharmacies provided by iPLATO has been implemented in London pharmacies, using texts to verify patients' smoking status and invite them to take part in smoking cessation services and follow-up treatment. Other health-related SMS-based systems are currently being implemented throughout low and middle income countries. In 2007, a program of text message reminders was being designed with a large teaching hospital in Johannesburg in an effort to make more efficient use of overworked healthcare workers [8]. SMS text messaging has also been highlighted as a preferred means of communication for mobilizing support and communicating during emergency and disaster 
situations [9]. Frontline SMS has developed a system using mass texting for surveys and community mobilization, which is free for non-governmental organizations (NGO) [10].

With mobile technologies, medical practitioners are able to instantly update and retrieve patients' records from anywhere within a telephone network coverage. This ensures timely update of patients' medical records. Physicians with up to date information are likely to make better prescription decisions. The adoption of mobile technology-based application could eliminate redundant paperwork, thereby facilitating more efficient and effective delivery of patient care. The objective of this paper is to provide a framework for voice-based medication adherence system. We also provide a review of the potential of mobile technologies for supporting medication adherence especially in outpatient settings. It provides instances of interventions provided via mobile technologies and its outcome measures.

\section{Related Works}

Many projects have applied the short message service (SMS) technology in ensuring patient adherence, such projects include: the Mobile Med Alert, a mobile medical alert system that sends SMS to patients, prompting them to take their drugs. It will design architecture for mobile health interventions and develop a prototype SMS-based system to improve out-patient adherence. Mobile Med Alert was developed using programming tools such as extensible hypertext markup language (xHTML), hypertext processor (PHP), MySQL and the integration of Ozeki SMS gateway. Its main features includes: it can alert patients about potential drug in-take at a scheduled time, in accordance to drug regimen; it allows for feedback mechanism whereby the Patient can respond appropriately to alert messages. In both cases, the application aims at increasing patients' compliance to treatment and in the long run, reduces the rate of noncompliance in relation to drug regimen [11].

Another research work considered a Mobile-phone based Patient Compliance System (MPCS) that can reduce the time-consuming and error-prone processes of existing self-regulation practice to facilitate self-reporting, non-compliance detection, and compliance reminder among patients in Nigeria. The uniqueness of this work is to apply social behavior theories to engineer the MPCS to positively influence patients' compliance behaviors, including mobile-delivered contextual reminders based on association theory; mobile triggered questionnaires based on selfperception theory; mobile enabled social interactions based on social construction theory, also explained how mobile phone can help patient to comply to their medication treatment; the existence of mobile phones and its uses in health sectors in Nigeria [12].

Also Projects such as WelTel have applied wireless technology in ensuring patient adherence: The WelTel Kenya1 was a multisite randomized clinical trial of HIV-infected adults initiating antiretroviral therapy (ART) in three clinics in Kenya. Patients were randomized (1:1) by simple randomization with a random number generating program to a mobile phone short message service (SMS) intervention or standard care. Patients in the intervention group received weekly SMS messages from a clinic nurse and were required to respond within 48hours. Randomization, laboratory assays, and analyses were done by investigators masked to treatment allocation; however, study participants and clinic staff were not masked to treatment. Primary outcomes were self-reported ART adherence ( $>95 \%$ of prescribed doses in the past 30 days at both 6 and 12 month follow-up visits) and plasma HIV-1 viral RNA load suppression (<400 copies per mL) 
at 12 months. The primary analysis was by intention to treat. Patients who received SMS support had significantly improved ART adherence and rates of viral suppression compared with the control individuals. Mobile phones might be effective tools to improve patient outcome in resource-limited settings [13].

In Norway, SMS messages are sent to educate parents with Type 1 diabetic children. These messages included definitions, facts and tips for managing diabetes [14]. Text to Change (South Africa) project employs an SMS-based quiz to test users' knowledge of HIV/AIDS and encourage testing and counseling [4]. Wedjat is a mobile medication reminder and monitoring system. It is a smart phone application designed to help remind its users to take the correct medicines on time and record the in-take schedules for later review by healthcare professionals [15].

The Uganda-Text to Change program aimed to encourage citizens to seek voluntary testing and counseling for HIV/AIDS. Multiple choice quizzes were administered through SMS to subscribers of celtel (Airtel). The program offered free airtime to users to encourage participation in the program. The quiz was interactive. When participants gave a wrong answer they received an SMS with the correct answer from the cell phone provider. The program motivated participants to go for voluntary testing and counseling at the local health center [4]. "South Africa-Aftercare" program by Cell-Life a non-governmental organization in Cape Town works with the public health system and health workers to collect patient information using mobile phones during home based care visits for HIV/AIDS patients receiving ART [16]. The solution is effective in that it eliminates the need for paperwork and enables the logging of accurate data on a large scale with minimum cost.

In [17], a Voice-based Mobile Prescription Application (VBMOPA) was designed and implemented to improve health care services. The application can be accessed anyplace anytime, anywhere through a mobile phone by dialing an appropriate number, this connects users to an eprescription application that is resident on a web server. This system could lead to costs and life savings in healthcare centers across the world especially in developing countries where treatment processes are usually cumbersome and paper based. Other projects include: TRICKs (Text Reminders for Immunization Compliance in Kids) which sends out text messages of immunization reminders prior to immunization dates [18]; Text messaging to motivate walking in older African-Americans [19]; Text4baby, the first free national health text messaging service in the United States that aims to provide timely information to pregnant women and new mothers to help them improve their health and the health of their babies [20]; a mobile phone text message program to measure oral antibiotic use and provide feedback on adherence to patients discharged from the emergency department [21] and the use of text messaging to increase the receipt of influenza vaccine among low-income urban children [22]. Studies have also been carried out to measure the effectiveness of electronic reminders, SMS, and phone calls in improving patient adherence [23-25].

HeartSaver, a mobile medical device for real-time monitoring of a patient's electrocardiogram (ECG) and automatic detection of several cardiac pathologies was developed in [26]. Another study investigated the potential of short message service (SMS) reminders at reducing nonattendance in physical therapy outpatient clinics. The primary outcome was rate of nonattendance without cancellation. Secondary outcomes were cancellation and attendance rates 
and exploration of other factors associated with nonattendance [27]. The Cameroon mobile phone SMS (CAMPS) trial; a randomized trial, tested the efficacy of weekly reminder and motivational text messages, compared to the usual care in improving adherence to Highly Active Antiretroviral Treatment in patients attending a clinic in Yaoundé, Cameroon [28]. Another study developed and tested MEMO; a mobile phone depression prevention intervention for adolescents. The study developed and tested the novel mobile phone delivery of a depression prevention intervention for adolescents [29].

\section{System Design and Implementation}

This paper introduces Voice MedAlert, a mobile medical alert system that calls and sends SMS to patients, reminding and prompting them to take their drugs. Patients receive alerts on drug intake methods, description and dosages, in order to ensure adherence to drug regimen and prescriptions via voice calls and text messages. Furthermore, patients can reply SMS alerts indicating whether they have taken the drugs or not. An incremental approach to software development is used for the development of the system.

The system consists of the voice user interface (VUI) design and modeled with Unified Modeling Language (UML). It was developed using tools such as HTML (Hypertext Markup Language), hypertext preprocessor (PHP), MySQL and the integration of the TWILIO REST API. Its main features includes: sending SMS messages to patients about potential drug in take at a scheduled time; sending SMS reminders to patients about appointments some hours before the scheduled appointment, placing voice calls across to patients to also remind them of scheduled appointments and dosing times. The system administrator enters information about prescription and appointments to be taken by the patients. The cron job checks the database every minute and if the prescription or appointment time conforms to the current system time, it then calls the scheduler which initiates the Twilio Rest API in sending SMS alert.

\subsection{VUI Design}

The VUI used for this system is the TWILIO Rest Web Service Interface. It is a voice application program interface (API) that allows you to query meta-data about your phone numbers, calls, text messages, and recordings. Since the API is based on REST principles, it's very easy to write and test applications. Its features include:

- Ability to send and receive SMS messages;

- Conversion of text to speech;

- Playing of audio files;

- Recording and storage of calls.

- It also enables you to create conference calls with up to 40 people in any one conference.

- In addition, Twilio rest has a speech to text engine that converts spoken words into text. 


\subsection{System Architecture}

The system is 3-tier client and server architecture as shown in Figure 1. It contains client devices, servers and a back-end running MySQL database.

- Client Devices: The client systems include VUI devices such as mobile phones (Personal Digital Assistant (PDA), cell phone and smart phone and other handheld communication devices).

- Servers: The server contains the Voice (TWILIO Rest Web Service Interface) and web server. The Twilio Rest contains the speech server (gateway) which has textto-speech (TTS) and voice browser. The web/application server stores the voicebased health-care content information. It uses HTTP to maintain Internet connectivity with the voice gateway.

- Databases: The database used is MySQL. It stores the information about appointments and prescriptions. MySQL is a server application (for database) able to carry out a great number of SQL commands.

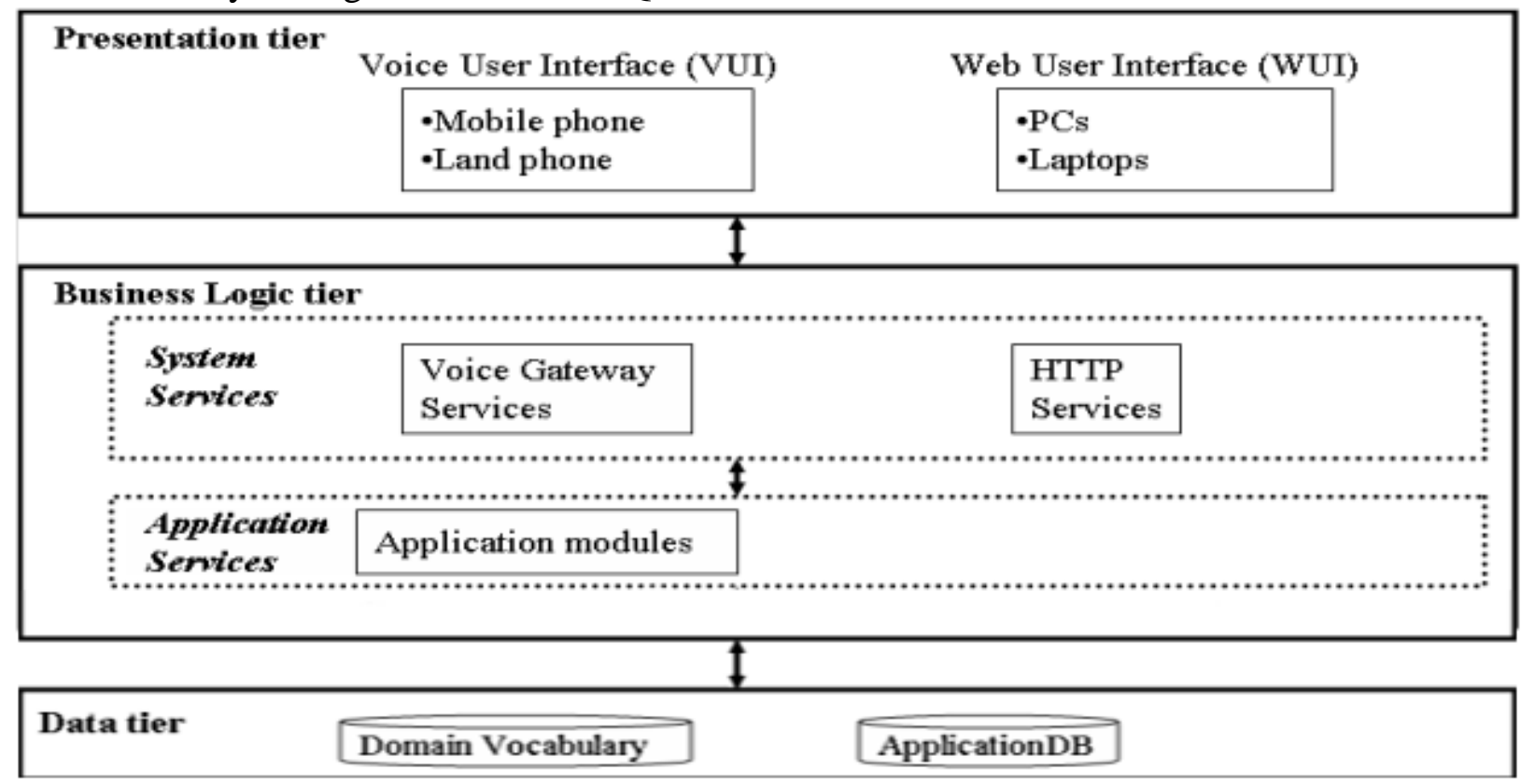

Figure 1. A Framework for Voice MedAlert.

\subsection{System Implementation}

The prototype was developed using programming tools such as extensible hypertext markup language (xHTML), Ozeki SMS gateway, TWILIO REST for the voice user interface (VUI), PHP for web user interface (WUI), Apache as middle-ware, and MySQL database as backend. The choice for these tools is because of their advantage as free and open source software [30]. The user interfaces for interacting with the system are shown in Figures 2 and 3. Figure 4 shows a screenshot of the text message on a mobile phone. 


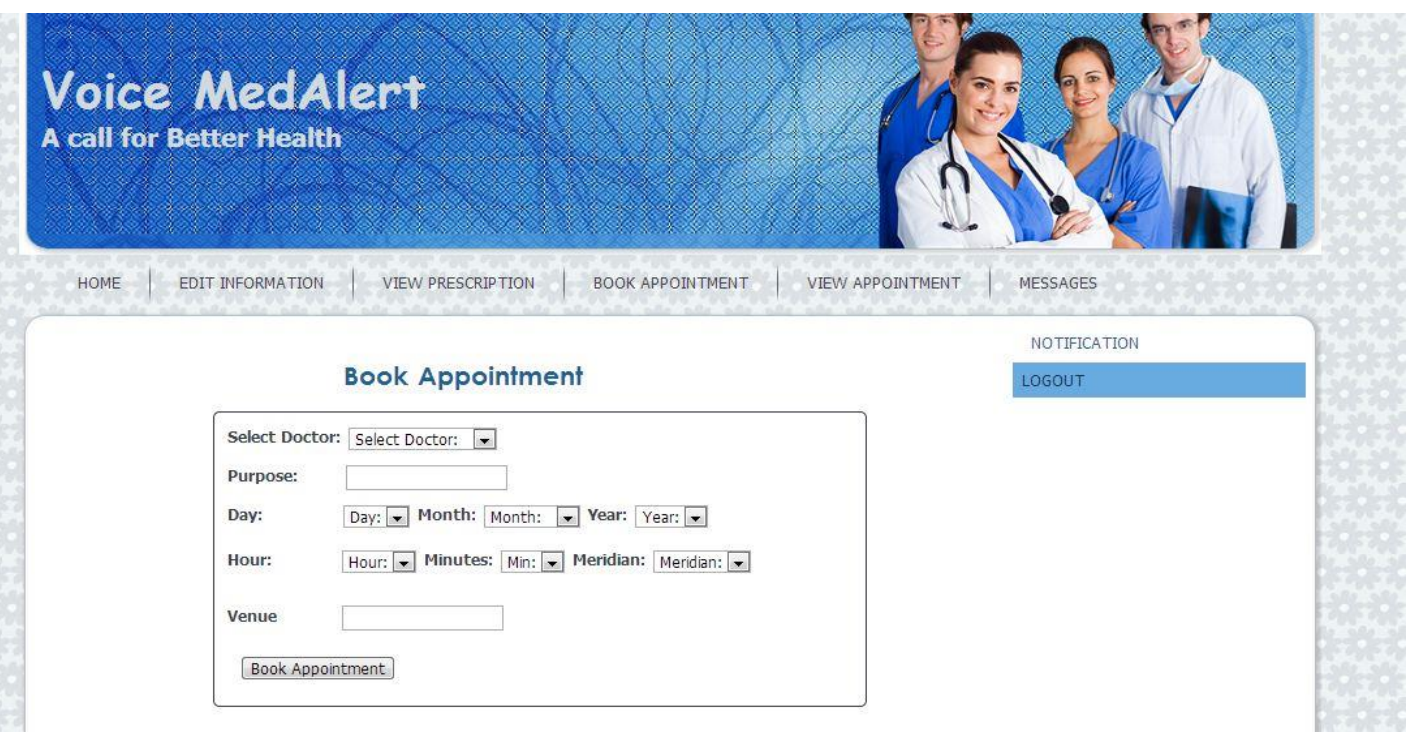

Figure 2: Screenshot of the "Book Appointment" Module of Voice MedAlert

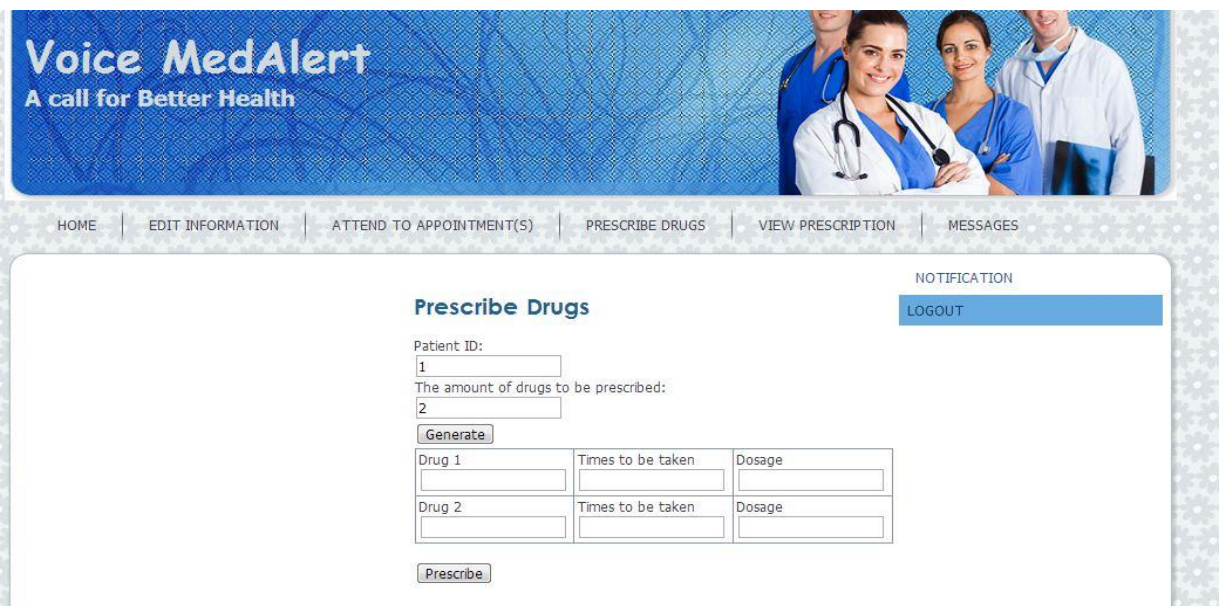

Figure 3: Screenshot of the "Prescribe Drugs" Module of Voice MedAlert

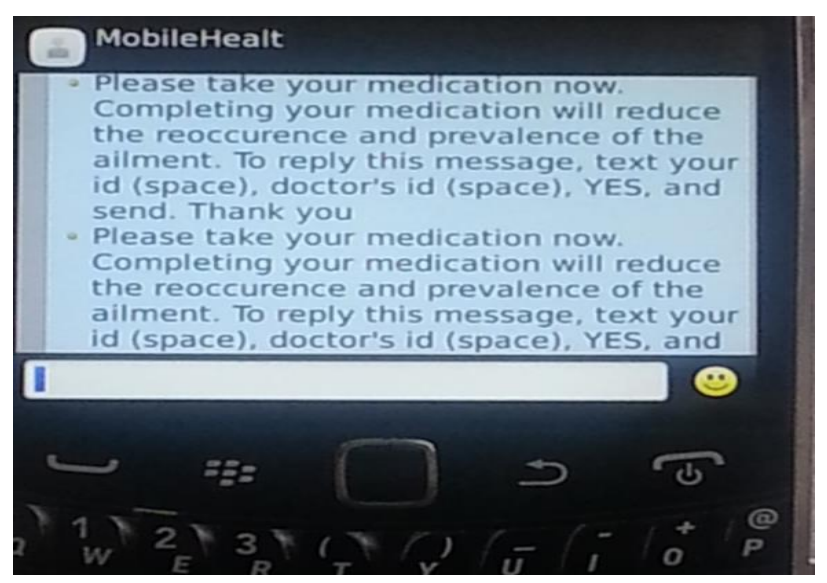

Figure 4: Screenshot of the Text Message on a Mobile Phone 


\section{Conclusion}

The mobile technology-based alert system discussed in this paper, allows patients receive SMS and voice alerts for appointments and medications. The work is in progress. The prototype is at the deployment and evaluation stage. The ubiquity of mobile phones and its current integration in health care has made it a worthy tool to this effect. The system will reasonably reduce humanto-human $(\mathrm{H} 2 \mathrm{H})$ contact (such as doctor to patient) by replacing it with human-to-system (H2S) interactivity. This system will lead to costs and life savings in healthcare centers' in developing countries where to ensure adherence to treatment, patients must walk many miles to clinics to receive and take medication and this is often not possible because of distance, lack of transportation, bad weather or a worsening condition that prevents them from leaving home.

The benefits of this intervention notwithstanding, it will not be a substitute for the patienthealthcare provider relationship. The extension and integration of the intervention into hospital information management systems (HIMS) will increase the potentials of such interventions in the provision of efficient and effective adherence strategies. This will make healthcare accessible and available to all - a giant step in the direction of the achievement of the MDGs on healthcare for all.

\section{Conflicts of Interest}

"The authors declare no conflict of interest".

\section{References and Notes}

1. Labiran A. Mafe, M. Onajole, B. and Lambo, E. (2008). Health Workforce country profile for Nigeria. $\quad$ http://www.hrhobservatory.afro.who.int/images/

Document_Centre/nigeria_country_profile.pdf.

2. Dictionary of Health Services Management. (1982) 2nd ed. Owing Mills, MD, National Health Publishing.

3. Osterberg L, Blaschke T. 2005. Adherence to medication. $N$ Engl J Med. 353(5), 487-97. PubMed http://dx.doi.org/10.1056/NEJMra050100

4. World Health Organization. (2003). Adherence to long-term therapies: Evidence for action. Geneva:Author. Available from www.who.int/chronic_conditions/adherencereport/en

5. http://telecomscompare.com/misc.cfm?type=News\&id=151 accessed $2^{\text {nd }}$ March, 2013

6. Kaplan WA. 2006. Can the Ubiquitous Power of Mobile Phones be used to Improve Health Outcomes in Developing Countries? Global Health. 2, $9 . \quad$ PubMed http://dx.doi.org/10.1186/1744-8603-2-9

7. The Economist (2006). A text a day, The Economist.

8. Mechael P. (2006). Exploring Health-related Uses of Mobile Phones: An Egyptian Case Study, Public Health \& Policy (p. 264). London: London School of Hygiene and Tropical Medicine

9. Association GSM. (2005). The Role of Mobiles in Disasters and Emergencies 1-34.London: GSM Association

10. Vital Wave Consulting. (2009). mHealth for Development: The Opportunity of Mobile Technology for Healthcare in the Developing World. United Nations. Retrieved from unpan1.un.org/intradoc/groups/public/documents/.../unpan037268.pdf 
11. Okuboyejo SR, Ikhu-Omoregbe NA, Mbarika VW. 2012. A Framework for the Design of a Mobile-Based Alert System for Outpatient Adherence in Nigeria. African Journal of Computing \& ICT. 5(5), 151-58.

12. Amosa BMG, Longe OL, Akinbode OP. 2012. Mobile-Phone Based Patient Compliance System for Chronic Illness care in Nigeria. Journal of Computer Science \& Technology. 12(1), 22-26.

13. Lester RT. Ritvo, P. Mills, E.J. Kariri, A. Karanja, S.et. al., (2010), "Effects of a Mobile Phone Short Message Service on Antiretroviral Treatment adherence in Kenya (WelTel Kenya1): A Randomized Trial", Lancet 2010; 376: 1838-45, DOI: 10.1016/S01406736(10)61997-6, www.thelancet.com.

14. Wangberg SC, Arsand E, Andersson N. 2006. Diabetes Education via Mobile Text Messaging. J Telemed Telecare. 12(1), 55-56. $\quad \underline{\text { PubMed }}$ http://dx.doi.org/10.1258/135763306777978515

15. Wang M, Tsai PH, Liu JWS, Zao JK. (2009), "Wedjat: A Mobile Phone Based Medicine Intake Reminder and Monitor," bibe, 423-430, in Proceedings of the Ninth IEEE International Conference on Bioinformatics and Bioengineering

16. Kinkade. S., Verclas, K. MobileActive.org. (2007). Wireless Technology for social change: Trends in Mobile Use by NGOs commissioned by UN foundation and Vodafone group foundation.

17. Ikhu-Omoregbe NA, Azeta AA. 2011. A Voice-based Mobile Prescription Application for Healthcare Services (VBMOPA). International Journal of Electrical \& Computer Sciences IJECS-IJENS. 10(02), 73-77. www.covenantuniversity.edu.ng.

18. Ahlers-Schmidt CR, Chesser AK, Nguyen T, Brannon J, Hart TA, et al. 2012. Feasibility of a Randomized Controlled Trial to Evaluate Text Reminders for Immunization Compliance in Kids (TRICKs). Vaccine. 30, 5305-09. $\quad \underline{\text { PubMed }}$ http://dx.doi.org/10.1016/j.vaccine.2012.06.058

19. Kim BH, Glanz K. 2013. Text Messaging to Motivate Walking in Older African Americans: A Randomized Controlled Trial. Am J Prev Med. 44(1), 71-75. PubMed http://dx.doi.org/10.1016/j.amepre.2012.09.050

20. Whittaker R, Matoff-Stepp S, Meehan J, Kendrick J, Jordan E, et al. 2012. Text4baby: Development and Implementation of a National Text Messaging Health Information $\begin{array}{lllll}\text { Service. } & \mathrm{Am} \quad \mathrm{J} \text { Public } & \text { Health. } & \text { 102(12), }\end{array}$ doi:http://dx.doi.org/10.2105/AJPH.2012.300736. PubMed

21. Suffoletto B, Calabria J, Ross A, Callaway C, Yealy DM. 2012. A Mobile Phone Text Message Program to Measure Oral Antibiotic Use and Provide Feedback on Adherence to Patients Discharged From the Emergency Department. Acad Emerg Med. 19(8), 949-58. PubMed http://dx.doi.org/10.1111/j.1553-2712.2012.01411.x

22. Kharbanda EO, Stockwell MS, Martinez RA, Vargas CY, Vawdrey DK, et al. 2012. Effect of a Text Messaging Intervention on Influenza Vaccination in an Urban, Low-Income Paediatric and Adolescent Population A Randomized Controlled Trial. JAMA. 307(16), 1702-08. PubMed http://dx.doi.org/10.1001/jama.2012.502

23. Wu AW, Snyder CF, Huang IC, Skolasky R, Jr, McGruder HF, et al. 2006. A randomized trial of the impact of a programmable medication reminder device on quality of life in patients with AIDS. AIDS Patient Care STDS. 20(11), 773-81. PubMed http://dx.doi.org/10.1089/apc.2006.20.773 
24. Chen Z. Fang, L. Chen, L. Dai, H. (2007). Comparison of an SMS text messaging and Phone Reminder to Improve attendance at a Health Promotion centre: A randomized controlled trial. Journal of Zhejiang University SCIENCE B, ISSN 1673-1581 (Print); ISSN 1862-1783 (Online), www.zju.edu.cn/jzus; www.springerlink.com

25. Wise J, Operario D. 2008. Use of Electronic Reminder Devices to Improve Adherence to Antiretroviral Therapy: A Systematic Review. AIDS Patient Care STDS. 22(6). doi:http://dx.doi.org/10.1089/apc.2007.0180. PubMed

26. Sankari Z, Adeli H. 2011. HeartSaver: A mobile cardiac monitoring system for autodetection of atrial fibrillation, myocardial infarction, and atrio-ventricular block. Comput Biol Med. 41(4), 211-20. PubMed http://dx.doi.org/10.1016/j.compbiomed.2011.02.002

27. Taylor NF, Bottrell J, Lawler K, Benjamin D. 2012. Mobile Telephone Short Message Service Reminders Can Reduce Nonattendance in Physical Therapy Outpatient Clinics: A Randomized Controlled Trial. Arch Phys Med Rehabil. 93(1), 21-26. PubMed http://dx.doi.org/10.1016/j.apmr.2011.08.007

28. Mbuagbaw L, Thabane L, Ongolo-Zogo P, Lester RT, Mills E, et al. 2011. The Cameroon mobile phone SMS (CAMPS) trial: a protocol for a randomized controlled trial of mobile phone text messaging versus usual care for improving adherence to highly active antiretroviral therapy. Trials. 12(1), 5. PubMed http://dx.doi.org/10.1186/1745-6215-12-5

29. Whittaker R, Merry S, Stasiak K, McDowell H, Doherty I, et al. 2012. MEMO-A Mobile Phone Depression Prevention Intervention for Adolescents: Development Process and Postprogramme Findings on Acceptability From a Randomized Controlled Trial. J Med Internet Res. 14(1), e13. PubMed http://dx.doi.org/10.2196/jmir.1857

30. Siemens G. (2003). Open source content in education: Part 2 - Developing, sharing, expanding resources, accessed April, 2007 from

http://www.elearnspace.org/Articles/open_source_part_2.htm. 